\title{
Aspectos innovadores de realidad virtual y sensores cinéticos para una mejora significativa utilizando el algoritmo de Fireworks para un juego Wii de un deporte colaborativo para una sociedad en una Smart City
}

\author{
Alberto Ochoa ${ }^{1}$, César Velázquez ${ }^{2}$, Juan Hernández ${ }^{1}$, Silvia Husted ${ }^{1}$, Diego Oliva ${ }^{3}$, \\ Anahí Solis ${ }^{1}$, Héctor Herrera ${ }^{1}$, José Mejia ${ }^{1}$, Saúl González ${ }^{1}$, Edna Cruz-Flores ${ }^{4}$ \\ ${ }^{1}$ Universidad Autónoma de Ciudad Juárez, Ciudad Juárez, Chihuahua, México \\ ${ }^{2}$ Universidad Autónoma de Aguascalientes, Aguascalientes, Ags., México \\ ${ }^{3}$ CUCEI-UDG, Guadalajara, Jalisco, México \\ ${ }^{4}$ Universidad Autónoma del Estado de Morelos, FCAeI, Cuernavaca, Morelos, \\ México \\ alberto.ochoa@uacj.mx
}

\begin{abstract}
Resumen. Cada cierto tiempo se publica un nuevo informe sobre la obesidad infantil. Los malos hábitos alimentarios y la vida cada vez más sedentaria de los niños en una sociedad fronteriza, ha provocado un aumento alarmante en los casos de niños con sobrepeso u obesos. Anteriormente, parecía un problema de países con hábitos alimentarios poco saludables, como los Estados Unidos o México en América Latina, donde la comida chatarra es parte de la dieta en la infancia. Sin embargo, la obesidad es un problema que ya tenemos a la vuelta de la esquina y que no es tan difícil combatir en los niños. En la presente investigación, el desarrollo de una aplicación que reduce el problema de la falta de movimiento en la infancia de una ciudad inteligente se considera un problema futuro, que es la principal contribución, junto con el logro de una forma innovadora de buscar un deporte olímpico sin la complejidad de trasladarse físicamente a un espacio con altos costos de mantenimiento y considerando las condiciones climáticas adversas.
\end{abstract}

Palabras clave: juegos basados en ejercicio virtual, cinética, sistema Wii, aplicaciones inteligentes en una smart city.

\section{Innovative Aspects of Virtual Reality and Kinetic Sensors for Significant Improvement using Fireworks Algorithm to a Wii Game of a Collaborative Sport to a Society in a Smart City}

\begin{abstract}
A new report on childhood obesity is published every so often. The bad habits of food and the increasingly sedentary life of children in a border society, has caused an alarming increase in the cases of children who are overweight or obese. Formerly it seemed a problem of countries with unhealthy eating habits, such as the United States or Mexico in LatinAmerica, where junk food is part of the diet in childhood. However, obesity is a problem that we already have around the corner and that is not so difficult to fight in children. In the present research the development of an application
\end{abstract}


that reduces the problem of the lack of movement in the childhood of a smart city is considered a future problem which It is the main contribution, coupled with achieving an innovative way of looking for an Olympic sport without the complexity of physically moving to a space with high maintenance costs and considering the adverse weather conditions.

Keywords: exergaming, kinetic, Wii system, smart applications in a smart city.

\section{Introducción}

El aumento de la obesidad infantil, un problema de gran importancia en una ciudad inteligente, determina los desafíos que se deben construir con respecto a las aplicaciones que involucran la Inteligencia Artificial. Los juegos de computadora para combatir la obesidad infantil son muy importantes para reducir un problema futuro en nuestra sociedad. Exergaming, juegos de computadora para ejercitar, los niños juegan cada vez menos en la calle y pasan más tiempo con los videojuegos y los juegos de computadora, por lo que llevan una vida más sedentaria. Esto, junto con los malos hábitos alimenticios, aumenta los casos de niños obesos cada año. ¿Qué pueden hacer los padres para evitar el sobrepeso en la infancia? Una apuesta que nos viene de la Universidad de Australia Occidental, la Universidad John Mores de Liverpool y la Universidad de Swansea en el Reino Unido es el exergaming, un anglicismo que viene de unir la palabra "exerdizze" en turco (ejercicio en inglés) con juegos (juego). Estos son juegos que ofrecen consolas como Xbox, Kinect o Wii en las que interactúas a través de la actividad física en las pruebas en las que tienes que correr, andar en bicicleta, jugar bolos o saltar vallas.

Los investigadores evaluaron a los niños que realizaron exergaming de alta y baja intensidad y midieron su gasto de energía. La conclusión a la que se llegó fue que el exergaming generó un gasto de energía comparable al ejercicio de intensidad moderada o baja, dependiendo de la dificultad del juego. Además, el juego fue satisfactorio para los niños, quienes disfrutaron de las actividades que realizaron.

Es una ventaja que los padres pueden aprovechar para evitar que los niños pasen tantas horas sentados frente a la consola, ya que se ha demostrado que puede obtener beneficios de salud a largo plazo. En cualquier caso, siempre debe ser uno de los medios que podemos utilizar para alentar a los niños a hacer alguna actividad física, pero no el único. Salir a la calle para jugar, correr, saltar, siempre debe estar en la agenda de los niños, como se muestra en la figura 1.

A. Emociones en los niños desde que surgió la psicología infantil basada en la ciencia en la segunda mitad del siglo XIX, se prometió proporcionar una base racional para la educación para el desarrollo general del niño. El desarrollo de toda esta área ofrece nuevas propuestas interesantes, como el uso del estudio de los niños, no solo para la psicología infantil, sino que también propone la creación de una ciencia enfocada al niño que va más allá de la psicología infantil [2]. Esto se lo agradece a William Preyer, quien es considerado el padre de la psicología infantil y el ojo no es el primero, pero si lo hizo con una precisión rigurosa, lo mismo se hace en sus observaciones de forma muy sistemática [1]. 


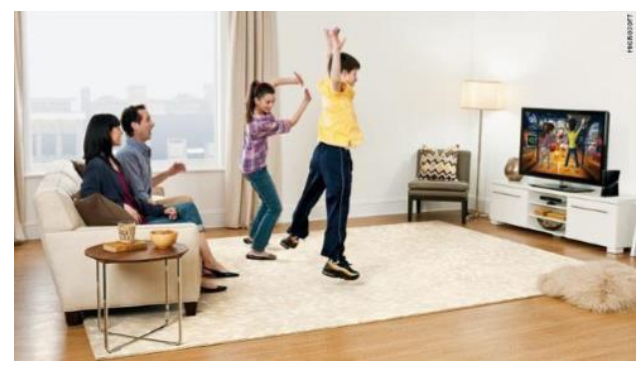

Fig. 1. Aplicación inteligente utilizando Kinect.

B. Trastornos emocionales en niños. Los trastornos mentales en los niños afectan a muchos niños y sus familias. Los niños de todas las edades, orígenes étnicos o raciales, y las regiones de los Estados Unidos tienen trastornos mentales. Según el informe del Consejo Nacional de Investigación y el Instituto de Medicina de la división (Prevención de trastornos mentales, emocionales y del comportamiento en jóvenes: progreso y posibilidades, 2009) que recopilaron los resultados de estudios anteriores, se estima que de 13 a 20\% de los niños que viven en los Estados Unidos (hasta 1 de cada 5) tiene un trastorno mental en un año determinado, y se gastan aproximadamente 247,000 millones de dólares al año en trastornos mentales infantiles [3]. En la literatura encontramos varias definiciones para referirse a problemas emocionales, mentales o de comportamiento. En estos días encontramos que se les conoce como "trastornos emocionales". El Acta de Educación para Personas con Discapacidades de la Ley de Educación ("IDEA" para abreviar) define el desempeño de los trastornos emocionales como "esa condición que exhibe una característica en mucho tiempo en un grado de nitidez que afecta negativamente al niño". Inmediatamente enumeramos las clasificaciones de los tipos de comportamientos como trastorno emocional [4]. a) La falta de habilidad para aprender que es inexplicable por razones intelectuales, sensoriales o de salud. b) La falta de capacidad para mantener relaciones personales en buenos términos con compañeros o con sus maestros. c) Comportamiento o sentimientos inconsistentes en circunstancias normales. d) Suele ser tristeza o depresión. e) Desarrollar síntomas físicos o miedos asociados con problemas personales o escolares. Hiperactividad: en este tipo de comportamiento se manifiesta al niño desatento, fácilmente distraído y con un grado de impulsividad. Asaltos: cuando el resultado del comportamiento termina en heridas, ya sea ellos mismos o a personas que los rodean. Retirada: la vida social muestra signos de retraso o la incapacidad de los individuos para relacionarse con su entorno. Incluye temores excesivos o ansiedad. Inmadurez: cuando experimenta episodios de llanto donde no está justificado; es incapaz de adaptarse a los cambios. Dificultades de aprendizaje: el aprendizaje no se desarrolla al mismo ritmo que el promedio en su entorno. Se mantiene por debajo del nivel por debajo de sus compañeros. Hay aún más niños pequeños con trastornos emocionales graves en los que el pensamiento se distorsiona, ansiedad severa, no son actos motores comunes y comportamientos demasiado irritables. A veces se diagnostica psicosis grave o esquizofrenia [4]

C. Terapia de juego: la terapia de juego se define como un modelo terapéutico de reconocimiento formal y también ha demostrado efectividad en niños con problemas de estrés emocional que contribuyen y se manifiestan en el niño durante el desarrollo 
normal. La terapia de juego se basa en el juego del niño como un medio natural de autoexpresión, experimentación y comunicación. Mientras el niño juega aprende sobre el mundo y sus relaciones, la realidad probada, explora las emociones y los roles. También le da al niño la posibilidad de externalizar su historia personal, liberando así sentimientos negativos y frustraciones, mitigando los efectos de experiencias dolorosas y que se estremezca, aliviando los sentimientos de ansiedad y estrés [5]. El terapeuta de juego es un especialista especializado y entrenado en el juego y adecuado para las diversas etapas de desarrollo profesional infantil, técnicas y métodos terapéuticos. Esto debe ser comprendido y entendido, así como participar en el juego del niño para poder crear una relación de confianza y confianza para la expresión y el manejo de los conflictos internos del niño. Además, la descarga y la comprensión de sus emociones están más atrapadas para que pueda reconocerlas y comprenderlas. Explorar temas que afectan sus vidas [6]. El juego serio propuesto no reemplaza la terapia lúdica de su terapeuta, sino que busca otorgar una herramienta de apoyo auxiliar y también es eficaz como apoyo para aquellos que están en contacto con niños en algún momento y muestran signos de un estado de ánimo bajo. Inclusive, los profesionales de la salud también podrían utilizarlo como parte de sus herramientas terapéuticas para controlar las emociones de sus pacientes.

D. Juegos serios: una antigua definición de los 70's que ha permanecido hasta ahora es que los juegos serios son aquellos que tienen un propósito expreso educativo o terapéutico y no solo divertidos. Dentro de esta definición es donde está el verdadero desafío para los desarrolladores porque mantener este equilibrio entre la diversión y el propósito del aprendizaje cuidadosamente planificado. Una vez que la construcción del juego se enfoca en el aprendizaje o el propósito terapéutico, sucede que la parte divertida de un zumo descuidado tan importante y necesario socava la calidad y el impacto del juego que debería tener en los usuarios. Las aplicaciones son variadas y van desde las más comunes a la educación, gestión, políticas, promoción, planificación, entre otras [7]. ¿Por qué juegos como soporte? La respuesta es simple e intuitiva, ya que son parte de la capacitación de las personas. Abarcan desde juegos individuales hasta juegos en computadoras, ya sea dos o tres o incluso miembros de muchos equipos. También un aspecto importante de la socialización y construcción de la identidad de los individuos. Cada cultura tiene juegos que se practican de generación en generación. Aunque siente que sus primeros juegos no fueron diseñados con propósitos de aprendizaje o terapéuticos, se puede ver que, además de la diversión y el entretenimiento para el individuo, ayuda en su desarrollo físico y mental porque no logran estimularlo. Y para reforzar lo anterior, el tiempo de la revolución es la tecnología que vivimos, donde prácticamente el uso de la tecnología se ha vuelto indispensable [8].

E. Lo importante a la hora de construir elementos de juegos serios. Dentro de una lista importante de características que incluyen un juego serio, debemos comenzar refiriéndonos a la memoria del Juego. Esta virtud es que el juego debe memorizar los logros o lo que ha aprendido el jugador, es que después del juego no solo se reinicia la dificultad del juego en el mismo punto, ya que esto puede hacer que el jugador no apela al juego porque lo hará ser el mismo para jugar de nuevo. Pero debe incluir una forma en que el juego recuerde lo que el jugador aprendió, debe ser educativo o apostar, saber qué parte del jugador de terapia se mantuvo así si el juego se utilizó 
como terapia de recuperación de algún tipo [9]. El flujo del juego es muy importante para sembrar la lealtad del jugador. Para ello debemos intentar modular la dificultad que no frustra ni aburre en el otro extremo para el usuario. Por esta razón, la dificultad debe ser modulada cuidadosamente [9]. Otra cosa muy importante es la historia o la narrativa. La combinación de historia, paisaje y contexto debe ser consistente con la cultura y el país. La narrativa es un elemento básico que debe indicar que el entorno gráfico debe ser inmersivo (sumergido, sumergido, escondido) para que el jugador sienta que está en el juego. Como la interactividad anterior debe estar presente para que el jugador interactúe tanto como sea posible jugando. No debemos olvidar también que deben proporcionar elementos coherentes con la realidad, ya que un objetivo del juego es que el jugador desarrolle y / o mejore sus habilidades. Otra de las cosas más importantes de un juego serio es proporcionar cierto grado de adaptabilidad, este es el juego que se adapta al estilo del jugador para que responda correctamente y, dependiendo del estilo de un juego serio, piense en el público quién jugará, las historias que puedan interesar, los entornos y los personajes con los que el jugador se sentirá atraído para jugar son una parte esencial del juego. Las reglas deben estar claramente definidas. El jugador. Y, sobre todo, no debemos dejar pasar un juego serio, por obligación debe ser divertido [8].

\section{Metodología de la propuesta}

Algoritmo de fuegos artificiales. Para cumplir con el tiempo, en la ausencia de un modelo de integración social, y políticas públicas, se ha incluido considerar el modelo de juego de depredador-presa para analizar las relaciones entre estas minorías y el resto del grupo mayoritario [3, 4]. En el marco de algoritmo de fuegos artificiales, cuando se encuentra un fuego artificial, una lluvia de chispas llenará el espacio local alrededor del fuego artificial $[1,2]$.

En nuestra opinión, el proceso de explosión de un fuego artificial se puede ver como una búsqueda en el espacio local alrededor de un punto específico donde se muestra el fuego artificial a través de las chispas generadas en la explosión. Cuando se nos pide que encontremos un punto $x j$ que satisfaga, ecuación (1):

$$
f(x j)=y \text {, }
$$

podemos activar continuamente "fuegos artificiales" en el espacio potencial hasta que una "chispa" apunte o esté bastante cerca del punto $x j$. Al igual que el proceso de activación de los fuegos artificiales, en la figura 2 se muestra un marco aproximado del FA. En el FA, para cada generación de explosión, primero se seleccionan y se publican, donde se disparan $n$ fuegos artificiales.

Luego, después de la explosión, se obtienen y se evalúan las fuentes de las chispas. Cuando se encuentra la ubicación óptima, el algoritmo se detiene. De lo contrario, en otras ocasiones, se seleccionan las cines y fuegos artificiales actuales para la próxima generación de explosiones. En la figura 2, se puede ver que el éxito del FA radica en un buen diseño, un proceso de explosión y un método adecuado para seleccionar las preferencias, que se detallan respectivamente en las siguientes subsecciones. 


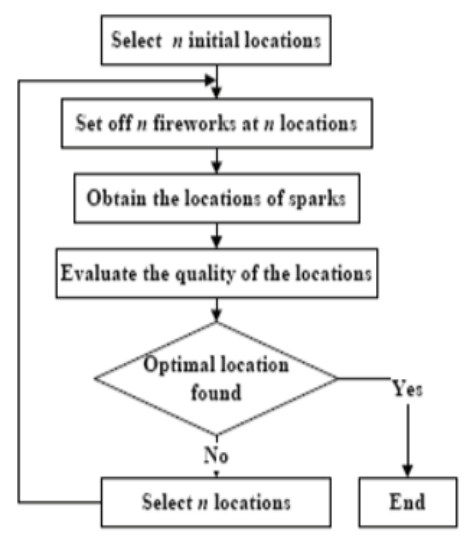

Fig. 2. Marco del algoritmo de fuegos artificiales.

Diseño de la explosión de fuegos artificiales. Al observar la exhibición de fuegos artificiales, hemos encontrado dos comportamientos específicos de explosión de fuegos artificiales. Cuando los fuegos artificiales están bien fabricados, se generan numerosas chispas, y las chispas centralizan el centro de la explosión. En este caso, disfrutamos de la espectacular exhibición de los fuegos artificiales. Sin embargo, para una mala explosión de fuegos artificiales, se generan muy pocas chispas, y las chispas se dispersan en el espacio. Las dos formas se muestran en la Figura 3. Desde el punto de vista de un algoritmo de búsqueda, un buen fuego artificial indica que el fuego artificial se ubica en un área prometedora que puede estar cerca de la ubicación óptima. Por lo tanto, es apropiado utilizar más chispas para buscar en el área local alrededor del fuego artificial. En contraste, un fuego artificial malo significa que la ubicación óptima puede estar lejos de donde se ubica el fuego artificial. Entonces, el radio de búsqueda debe ser mayor. En el FA, se generan más chispas y la amplitud de la explosión es menor para un buen fuego artificial, en comparación con uno malo [11].

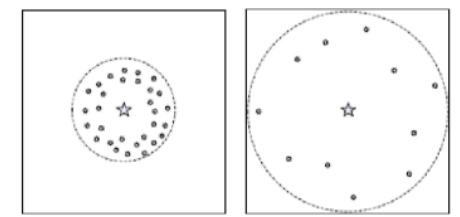

(a) Buena explosión / (b) Mala explosión

Fig. 3. Dos tipos de explosión de fuegos artificiales.

Número de chispas. Supongamos que el FA está diseñado para el problema general de optimización, ecuación (2):

$$
\text { Minimize } f(x) \in \mathbb{R}, x_{\min } \leqslant x \leqslant x_{\max },
$$

donde $x=x 1, x 2, \ldots, x d$ denota una ubicación en el espacio potencial, $f(x)$ es una función objetivo, y xmin y xmax denotan los límites del espacio potencial. Luego, el número de chispas generadas por cada $x i$ de fuego artificial, ecuación (3), se define de la siguiente manera: 


$$
s_{i}=m \cdot \frac{y_{\max }-f\left(\boldsymbol{x}_{\boldsymbol{i}}\right)+\xi}{\sum_{i=1}^{n}\left(y_{\max }-f\left(\boldsymbol{x}_{\boldsymbol{i}}\right)\right)+\xi},
$$

donde $m$ es un parámetro que controla el número total de chispas generadas por los $n$ fuegos artificiales, $y \max =\max (f(x i))(i=1,2, \ldots, n)$ es el valor máximo (peor) de la función objetivo entre los $n$ fuegos artificiales, y $\xi$, que denota la constante más pequeña en la computadora, se utiliza para evitar el error de división cero. Para evitar los efectos abrumadores de los espléndidos fuegos artificiales, los límites se definen para sí, como se muestran en la ecuación (4).

$$
\hat{s}_{i}= \begin{cases}\operatorname{round}(a \cdot m) & \text { if } s_{i}<a m \\ \operatorname{round}(b \cdot m) & \text { if } s_{i}>b m, a<b<1, \\ \operatorname{round}\left(s_{i}\right) & \text { otherwise }\end{cases}
$$

donde $a$ y $b$ son parámetros const. Amplitud de explosión. En contraste con el diseño del número de chispas, la amplitud de una buena explosión de fuegos artificiales es menor que la de una mala. La amplitud de explosión para cada fuego artificial se define de la siguiente manera, ecuación (5):

$$
A_{i}=\hat{A} \cdot \frac{f\left(x_{i}\right)-y_{\min }+\xi}{\sum_{i=1}^{n}\left(f\left(\boldsymbol{x}_{\boldsymbol{i}}\right)-y_{\text {min }}\right)+\xi},
$$

donde $A^{\wedge}$ denota la amplitud máxima de explosión, y ymin $=\min (f(x i))(i=1,2, \ldots$, $n$ ) es el valor mínimo (mejor) de la función objetivo entre los $n$ fuegos artificiales. Generando chispas. En una explosión, las chispas pueden sufrir los efectos de una explosión desde direcciones $z$ aleatorias (dimensiones). En el FA, obtenemos el número de direcciones afectadas al azar de la siguiente manera, ecuación (6):

$$
z=\operatorname{round}(d \cdot \operatorname{rand}(0,1))
$$

donde $d$ es la dimensionalidad de la ubicación $x$, y rand $(0,1)$ es una distribución uniforme sobre $[0,1]$. La ubicación de una chispa del fuego artificial xi se obtiene utilizando el algoritmo 1. Al simular el proceso de explosión, se genera primero la ubicación de una chispa $x x$. Luego, si la ubicación obtenida se encuentra fuera del espacio potencial, se asigna al espacio potencial de acuerdo con el algoritmo.

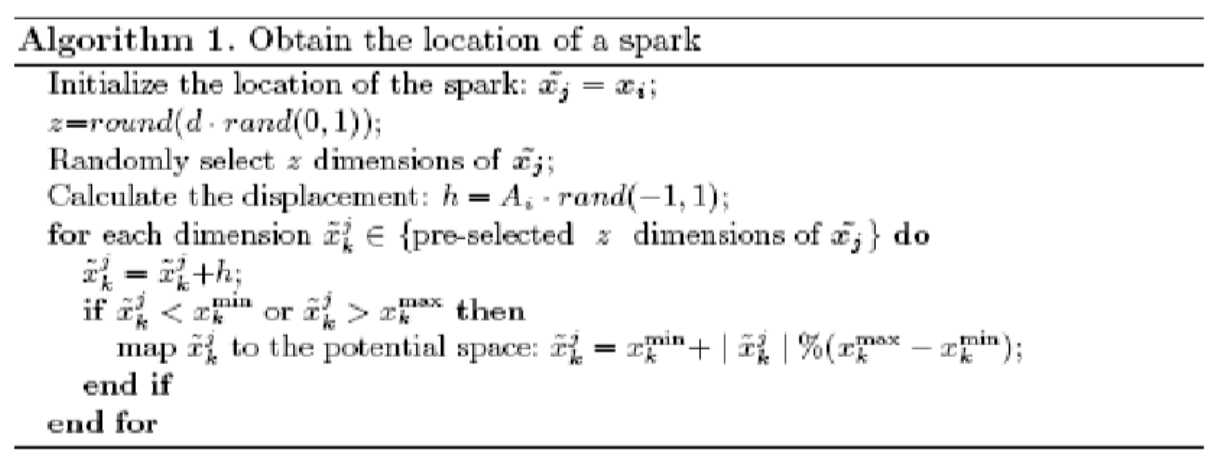

Para mantener la diversidad de chispas, diseñamos otra forma de generar chispas: la explosión gaussiana, que se muestra en el algoritmo 2. Se utiliza una función 
gaussiana $(1,1)$, que denota una distribución gaussiana con media 1 y desviación estándar 1, para Definir el coeficiente de la explosión. En nuestros experimentos, se generan $\hat{m}$ chispas de este tipo en cada generación de explosión.

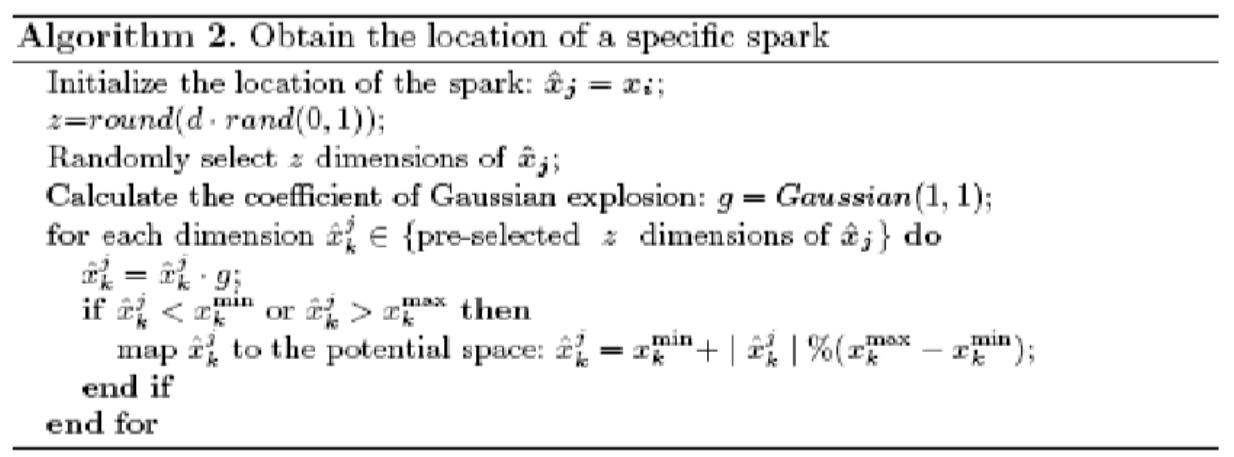

Selección de ubicaciones. Al comienzo de cada generación de explosión, se deben seleccionar $n$ ubicaciones para la explosión de fuegos artificiales. En el FA, la mejor ubicación actual $x$, sobre la cual la función objetivo $f(x *)$ es óptima entre las ubicaciones actuales, siempre se mantiene para la siguiente generación de explosión. Después de eso, se seleccionan $n-1$ ubicaciones en función de su distancia a otras ubicaciones para mantener la diversidad de chispas. La distancia general entre una ubicación $x i$ y otras ubicaciones se define de la siguiente manera:

$$
R\left(x_{i}\right)=\sum_{j \in K} d\left(x_{i}, x_{j}\right)=\sum_{j \in K}\left\|x_{i}-x_{j}\right\|,
$$

donde $K$ es el conjunto de todas las ubicaciones actuales de fuegos artificiales y chispas. Entonces, la probabilidad de selección de una ubicación xi. (ecuación (8)). Se define de la siguiente manera:

$$
p\left(x_{i}\right)=\frac{R\left(x_{i}\right)}{\sum_{j \in K} R\left(x_{j}\right)} .
$$

Al calcular la distancia, se puede utilizar cualquier medida de distancia, incluida la distancia de Manhattan, la distancia euclidiana, la distancia en ángulo, etc. [9]. Cuando $d(x i, x j)$ se define como $|f(x i)-f(x j)|$, la probabilidad es equivalente a la definición de la probabilidad basada en la densidad inmune en [10].

\section{Prototipo propuesto}

Debido al clima existente en Ciudad Juárez - Una sociedad fronteriza - donde la temperatura durante más de tres meses no permite actividades al aire libre, y se practican pocos deportes además del béisbol y el fútbol, para nuestra investigación decidimos elegir el waterpolo. Los estudiantes de los niveles escolares básicos aceptan colaborar en equipo, respetar las reglas y que el juego pueda interconectarse con otros usuarios en un modelo llamado "Modelo cooperativo para la resolución de problemas", una idea innovadora propuesta en el rol masivo de jugadores múltiples en línea. Los juegos (MMORPG). La cooperación entre jugadores para realizar tareas 
difíciles es a menudo una mecánica integral del juego, y surgen grupos organizados de jugadores, a menudo llamados gremios, clanes o facciones. A veces las relaciones con los jugadores dentro del juego se extienden a amistades o relaciones románticas en el mundo material. En otros casos, los socios románticos y los grupos de amigos del mundo material encuentran que jugar juntos fortalece sus vínculos. Utilizamos componentes comprados con nuestro Proyecto para desarrollar nuestro modelo innovador como se muestra en la figura 4.

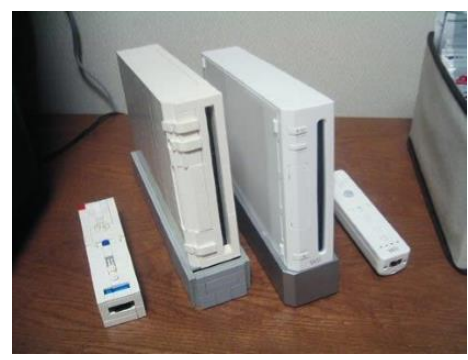

Fig. 4. Componentes de la aplicación Wii desarrollados.

La idea principal del Juego serio para una plataforma Wii es desarrollar las habilidades asociadas con la empatía y las habilidades psicomotoras para mejorar el rendimiento de los niños, es por eso que, para construir el prototipo del juego serio, inicialmente utilizamos tres escenarios asociados con la práctica del Waterpolo: una piscina cubierta, una piscina olímpica o un área delimitada en un parque acuático. Al comenzar nuestro juego serio, cada jugador debe registrar su nombre y la posición asignada para que el hardware refleje los movimientos necesarios para jugar en equipo. Y determine la modalidad del juego, en este caso, el jugador seleccionará un nivel que va de simple a complejo. En el nivel más simple, el jugador tendrá la posibilidad de verificar si los movimientos realizados son apropiados de acuerdo con el regalo de FINA, es por eso que la importancia de nuestra propuesta radica en que, junto con jugar simultáneamente con otros jugadores, puede aprender de una manera más apropiada. El juego irá demostrando que puede completar los desafíos propuestos $\mathrm{y}$, a su vez, desarrollar sus habilidades y conocimientos en el Waterpolo, es por eso que Serious Game propone al usuario demostrar sus habilidades y conocimientos, como el juego serio progresa cada vez que el jugador logra los objetivos deseados, se crea un modelo de sonido para recompensar el buen desempeño en el juego serio, como se muestra en la figura 5.

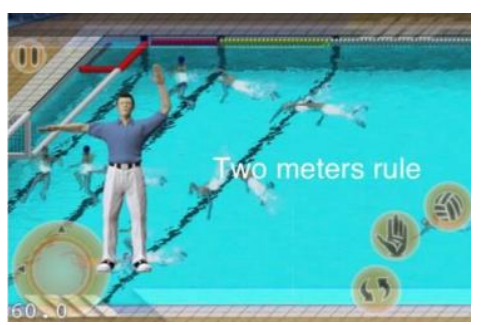

Fig. 5. Nuestro Wii Serious Game Model utilizando una tarea de colaboración para jugar Waterpolo. 
Como se menciona en este documento, este proyecto se encuentra actualmente en la fase de construcción. Este prototipo Serious Play tiene la firme intención de tomar la siguiente fase de construcción completa y funcional. Una fundación patrocinada por uno de los hospitales de niños más importantes de la ciudad de Paso Texas en los Estados Unidos. Ha mostrado un interés genuino en el proyecto, por lo que mencionó que proporciona el apoyo necesario para su realización. Esta fundación cuenta con programas de cuidado infantil y cuenta con estrategias interesantes y muy eficientes. Es por eso que le gustaría tener este tipo de herramientas integradas en sus programas.

Para esto, pronto será formalizado por ambas partes comprometidas a apoyar el proyecto que se muestra en este documento. En futuras investigaciones, tratamos de modificar un juego basado en el trabajo colaborativo en un grupo (estamos eligiendo rugby seven) con una alta intensidad de presión para cada niño y modificamos la importancia relacionada con el soporte de este tipo de presión relacionada con la responsabilidad de una actividad colectiva, una aproximación se relacionará con lo que implica Waterpolo, como se muestra en la figura 5.

Un aspecto muy relevante, es considerar que si alguien pregunta por qué le gusta usar nuestro Juego serio, este usuario podrá responder: porque ha tenido un ámbito lúdico y una selección adecuada con el avatar, por lo que podría tener empatía por nuestra propuesta.

Al analizar con más detalle el grupo de personas que usaron nuestros Serious Games, determinamos que, como el juego de roles, es un pasatiempo que los une y les brinda oportunidades para ayudarse mutuamente y a su comunidad de videojuegos. Es un entorno seguro en el que puede experimentar interacciones sociales, algo fundamental cuando el clima no lo permite

Este grupo de usuarios de nuestro Juego serio dice que han sido testigos del crecimiento personal de los individuos en términos de su autoestima y la expansión de sus interacciones sociales como resultado del juego. Este es solo uno de los beneficios del juego.

Nuestra investigación mostró que se descubrió que todos pueden encontrar algunas horas a la semana para "salvar el universo, atrapar a los villanos o resolver misterios", incluido aprender a practicar waterpolo, y jugar con la computadora es tan divertido como cualquier otra actividad en nuestra investigación. Jugar nuestro juego serio puede fortalecer una variedad de habilidades como matemáticas y leer recomendaciones en línea.

Aumente la capacidad de pensar y hablar de manera clara y concisa, al formular e implementar sus planes, cooperar y comunicarse con otros, así como aumentar la capacidad de análisis. Información escrita y verbal. Colocado en el mercado, nuestro juego serio determinará que los jugadores sean miembros cohesivos del grupo en los juegos multijugador, pueden ayudar a las personas a desarrollar habilidades de liderazgo y promover la cooperación, el trabajo en equipo, la amistad y la comunicación abierta.

En otros estudios relacionados con este tipo de Wii Serious Games, tratamos de compararlos con nuestros colegas de Montenegro que proponen y desarrollan un juego serio innovador que involucra un modelo para los practicantes de esgrima. Este deporte está alcanzando una gran popularidad en esta sociedad. En la siguiente figura 6 se puede mostrar un modelo representativo: 


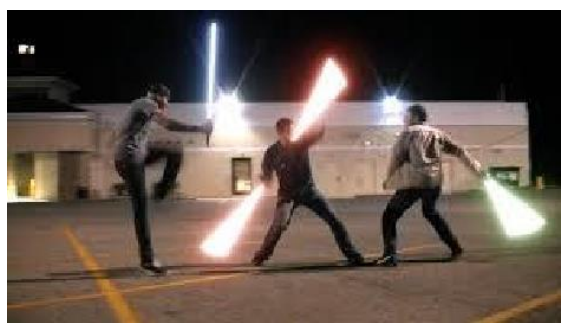

Fig. 6. Uso del software de Wii para mejorar el rendimiento en el Pentatlón moderno.

¿Qué esperaban los usuarios de nuestra propuesta de Juego Serio en este modelo de Wii para aprender y practicar el Waterpolo? Mejore el estado de ánimo del jugador según el juego transcurrido a través de las herramientas que se utilizarán como música de fondo, utilizando técnicas de musicoterapia para animar a los espíritus Jugador [11]. Otro elemento de la estrategia son los colores del escenario. A través del efecto del color en el estado de ánimo es tener al jugador en un estado emocional positivo [10]. El tercer elemento es a través de los sonidos en el juego. Con cada éxito y cada falla, el entorno ambiental suena el escenario en el que estás jugando. Y el cuarto elemento es el reconocimiento de los logros. A través de insignias, medallas, trofeos, puntuaciones y citas, usted desea que el jugador se sienta satisfecho con el reconocimiento de cada logro [12].

\section{Resultados}

Las escenas estructuradas asociadas con los agentes no se pueden reproducir en general, ya que solo representan un momento dado en el espacio y el tiempo de las diferentes sociedades. Estos representan una forma única e innovadora de comportamiento adaptativo que resuelve un problema computacional que no trata de agrupar las sociedades solo con un factor asociado con su apariencia externa (fenotipo), tratando de resolver un problema computacional que involucra un cambio complejo entre las relaciones existentes con la población que practican estos deportes. Las configuraciones generadas pueden relacionarse metafóricamente con el conocimiento del comportamiento de la comunidad con respecto a un problema de optimización (para ajustarse al clúster social y culturalmente con otras personas similares, sin ser del mismo deporte [4]). En la tabla 1 se muestra una muestra de siete deportes que describen las características analizadas en cada deporte diferente y su práctica requerida. Intentamos mejorar la siguiente ecuación 4 , en esta descripción, $K$ es el desempeño de la práctica de cada deporte en la ciudad y sus respectivas promociones en diversos lugares. (ecuación 9).

$$
K=[C+C S+G A L] \pm C B S,
$$

en donde: $C=$ representa si el deporte se puede practicar en cualquier lugar, por ejemplo, el Ajedrez. $C S=$ representa el capital simbólico asociado con la perspectiva de una sociedad, la práctica de esgrima se considera sofisticada. $G=$ es el género del rango de la población, en la Ciudad de Juárez la pirámide de la población es diferente al resto de América Latina porque la violencia provoca muertes y el éxodo de la 
ciudad. $A L=$ Aspecto lúdico relacionado con la edad de los niños que practican un deporte, por ejemplo, en el Trampolín, el aspecto lúdico se asocia con la alta promoción de este deporte. $C B S=$ Beneficios sociales: los costos relacionados con cada deporte, en el caso de la gimnasia rítmica, se asocian con la mejora de la salud en los niños sedentarios menos peso porque requieren muchas actividades deportivas relacionadas con los cuatro eventos: pelota, garrote, aro y cinta.

Tabla 1. Análisis multivariable con la información del número de espacios a recuperar para practicar estos deportes virtuales y sus representaciones por género (problema) -valores a masculino y femenino respectivamente-, el estado social por su práctica, divertido de usar la aplicación Wii, el clima, mejora el aumento de la salud para cambiar los paradigmas al sedentarismo en los niños y, finalmente, la relación entre el costo / beneficio social asociado con su práctica.

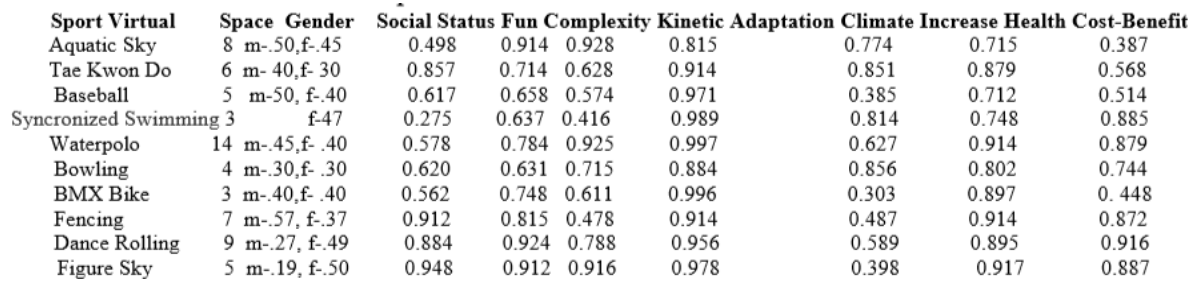

Hasta la fecha, se está desarrollando una Herramienta para la toma de decisiones para identificar en forma gráfica la forma en que la violencia afecta un índice compuesto al IDH en diferentes partes de la ciudad y con diferentes escenarios según la perspectiva de aumento o disminución del mismo. Para estos tres escenarios se están construyendo (progreso optimista, pesimista y gradual) para la identificación de cambios con respecto a la temporalidad y la ubicuidad de la información. El análisis e interpretación de estos escenarios con respecto al tiempo debe tratarse con sumo cuidado, buscando hacer un balance entre la predicción numérica realizada y justificada por el uso de ecuaciones, pudiendo seleccionar un índice, un índice compuesto, un factor o un rango asociado. La figura 7 muestra la perspectiva de la Herramienta de toma de decisiones propuesta y sus componentes principales, incluido un generador de escenarios asociado con el año de predicción numérica, que puede mostrarse mediante el uso de gráficos de radar y tacómetros de variabilidad estadística, los cambios en los escenarios mostrados, el ajuste de parámetros con respecto a los aspectos sociales y su relación con el año a proyectar, la visualización de un mapa que muestra en color los cambios en el IDH para cada AGEP de la ciudad asociada con el aumento en la violencia y la visualización de un guion narrativo que detalla los parámetros finales más importantes de la simulación realizada, como se puede ver en la figura 7 .

Uno de los aspectos más relevantes de la investigación realizada es la migración de la herramienta de toma de decisiones al sitio del Centro de Investigación Social de la UACJ, de modo que cualquier usuario pueda consultar con respecto a los indicadores asociados a HDI y su efecto de la Violencia existente en la ciudad. Uno de los aspectos metodológicos que justifican los escenarios para su simulación, se asocia con las encuestas de percepción de violencia que se han llevado a cabo en el Centro de Investigación Social y que muestran las variables asociadas con diversos elementos del estado que la sociedad mantiene con respecto a los indicadores de violencia más 
prevalentes. Otro aspecto que se desarrollará será la implementación de dicha Herramienta de toma de decisiones en dispositivos móviles como se propone en [11], que tienen la capacidad de modificar valores en situaciones de alta incertidumbre, como el tipo de cambio en el dólar mexicano. -prelación de la relación con la economía y en la parte social, el aumento de la violencia en un mes específico -en esa categoría, la disminución de las muertes durante 2011 puede estar asociada con el aumento de la extorsión, el secuestro y el robo con violencia.

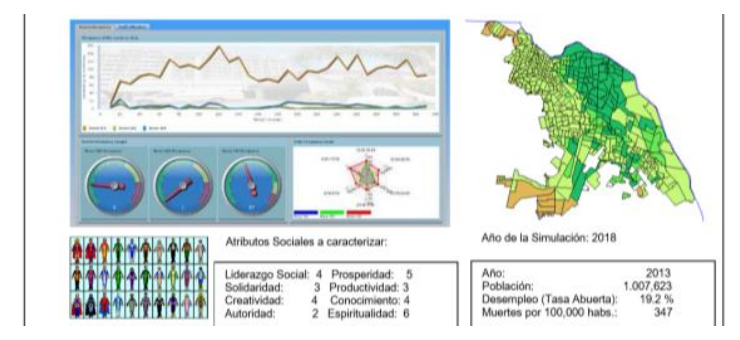

Fig. 7. Simulador de la Herramienta de Toma de Decisiones.

\section{Discusiones e investigación futura}

El aspecto más relevante de esta investigación es consolidar la práctica de deportes en niños y jóvenes que, debido a las condiciones climáticas, no pueden ponerse de acuerdo para unirse para practicar un deporte junto con la confianza en sí mismos que generan los jugadores, lo que permite mejorar su desempeño en otras áreas de su vida diaria a través de un modelo de apoyo emocional en los niños, lo que conlleva un compromiso y una complejidad intrínseca en su desarrollo motor. Teniendo en cuenta que la infancia es una etapa vulnerable en la que también se encuentran en pleno desarrollo y cualquier evento o suceso puede causar efectos negativos y puede dejar al niño marcado permanentemente por el resto de su vida [13]. Por lo tanto, es muy importante centrarse más en cómo obtener resultados asociados con el desempeño del grupo, patrocinado por el individuo. Es por eso que el futuro del Juego serio requerirá una investigación más profunda que permita tener un gran impacto al tener la oportunidad de ayudar a los niños y jóvenes que no tienen acceso a los deportes por varias razones. En el futuro, los Juegos serios pueden presentar una oportunidad natural debido en gran parte a la aceptación de que los videojuegos tienen en esta era y más aún con la ventaja de que estas generaciones tienen fácil acceso a la tecnología. Como se mencionó en la presente investigación, este prototipo desea continuar la consolidación para establecer diversos equipos asociados principalmente con el avatar. Este prototipo en lo que respecta al Juego Serio para lograr la fase de construcción completa y funcional. En este momento y debido a un proyecto con financiamiento de la Unión Europea y la colaboración de FINA, queremos hacer una aplicación Wii que permita ser inclusivo a través de estrategias interesantes y muy eficientes asociadas con la movilidad. FINA está muy interesada en este tipo de aplicaciones que podrían diversificar la práctica del waterpolo en los países en desarrollo. En futuras investigaciones, tratamos de mejorar la práctica de un deporte asociado con un juego y en base al trabajo colaborativo en un grupo con alta 
intensidad de presión para cada niño, como lo es el tenis profesional, y modificamos la importancia relacionada con el apoyo de este tipo de presión relacionado con la responsabilidad de una actividad colectiva [14], como se muestra en la figura 8.

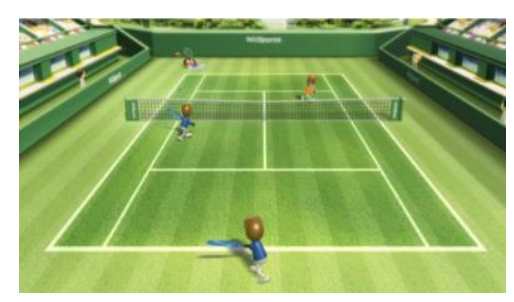

Fig. 8. Un juego serio basado en actividades colectivas y relacionado con el aumento de habilidades sociales.

El experimento principal consistió en detallar cada uno de los 47 deportes, con 500 agentes y una condición de desempleo de 50 generaciones, lo que nos permitió generar diferentes escenarios relacionados con los horizontes temporales, que se obtuvieron después de comparar diferentes similitudes culturales y sociales en cada comunidad, y para determinar las relaciones existentes entre cada uno en relación con la Distancia de Mahalanobis (el número de puntos que indica cada deporte y el tamaño de las personas representa el número de personas que determinan la magnitud relacionada con la sociedad). Diseño propuesto de experimentos.

Por otra parte, los objetivos específicos se determinarán a través de un estudio correlacional, en el que experimentará con diferentes variables. Esto con el propósito de conocer la relación existente entre dos o más variables para comprender qué variables son las que más afectan el IDH, como; el diseño de políticas públicas que mejoren la calidad de vida de las personas con problemas como la inseguridad, la violencia y el secuestro. Asimismo, se pretende analizar el grado de asociación entre estas variables. Estos resultados serán arrojados a través de un diseño factorial que intentará analizar mediante un diseño de experimentos con arreglo ortogonal, el efecto que las diferentes variables independientes tienen como violencia, inseguridad y secuestro en diferentes niveles como edad, género y nivel socioeconómico, aproximadamente la variable dependiente que, en nuestra investigación, es un efecto asociado con la calidad de vida denominada HDI, o que, en su caso, no está presente. En la presente investigación, se propone un diseño de experimentos en el que se llevarán a cabo diferentes interacciones de las variables a estudiar: violencia, inseguridad, extorsión y secuestro, que son las variables de control (Figura 9). Asimismo, estas variables se estudiarán en cuatro niveles: área geográfica, nivel de estudios, género y edad, que son las variables de ruido.

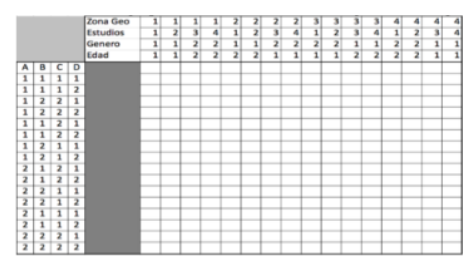

Fig. 9. Diseño de experimentos propuestos. 
Es importante determinar cambios paradigmáticos en nuestro modelo y justificar que se necesita estimar un costo/beneficio social.

\section{Referencias}

1. Jaeger, S.: Origins of Child Psychology: William Preyer, en W.R. Woodward. Woodward: s.n., pp. 300-321 (1982)

2. Chrisman, O.: Paidologie; Entwurf zu einer Wissenschaft des Kindes. s.1.: Jena, Bernhard Vopelius (1896)

3. HHS of USA. Center for Disease Control and Prevention. Especiales de los CDC: Salud mental de los niños: Apoyo de los servicios de salud conductual para quienes los necesitan http://www.cdc.gov/spanish/especialesCDC/ SaludMentalNinos/index.html

4. U.S. Department of Education of USA. Center for Parents Information and Resources. Sobre la Ley IDEA. http://www.parentcenterhub.org/repository/ sobreidea/

5. Tómas, U.: Elpsicoasesor. http://elpsicoasesor.com/25-poderes-terapeuticos-del-juego/

6. Schaefer, C.E.: Terapia de Juego. México: Editorial El Manual Moderno,S.A. de C.V. (2012)

7. Braga, P.H.C., Silveira, I.F.: A Pattern Language for semi-automatic generation of Digital Animation through hand-drawn Storyboards. Programa de Pós Graduação em Engenharia Eléctrica e Computação (2015)

8. Silveira, I.F.: Juegos Serios. Brazil: UACJ, Juegos Serios: teoría y práctica (2016)

9. Csíkszentmihályi, M.: Flow - The Psychology of optimal experience. New York: Harper \& Row (1990)

10. Heller, E.: Psicología del color: Como actúan los colores sobre los sentimientos y la razón. s.1.: Rústica (2016)

11. Lopez, J.J.B.: Musicoterapia como alternativa terapéutica en la depresión. Guatemala: Universidad de San Carlos de Guatemala (2012)

12. Leary, M.E.: Interpersonal Rejection. New York: Oxford University Press (2001)

13. The Hospital for Sick Children. About Kids Health. Efectos psicológicos del desastre sobre los niños. http://www.aboutkidshealth.ca/En/HealthAZ/ Multilingual/ES/Pages/psychological-effects-of-disaster-on-children.aspx

14. Garcia-Mundo, L., Vargas-Enriquez, J., Genero, M., Piattini, M.: ¿Contribuye el Uso de Juegos Serios a Mejorar el Aprendizaje en el Área de la Informática? Actas de las XX JENUI, pp. 303-310 (2014) 